\title{
Oxidative Stress in Hypobaric Hypoxia and Influence on Vessel-Tone Modifying Mediators
}

\author{
Jacqueline Pichler Hefti, ${ }^{1,2}$ Denise Sonntag, ${ }^{3}$ Urs Hefti, ${ }^{4}$ Lorenz Risch, ${ }^{5}$ Otto D. Schoch, ${ }^{6}$ \\ Alexander J. Turk, ${ }^{7}$ Thomas Hess, ${ }^{8}$ Konrad E. Bloch, ${ }^{7}$ Marco Maggiorini, ${ }^{9}$ \\ Tobias M. Merz, ${ }^{10}$ Klaus M. Weinberger, ${ }^{3}$ and Andreas R. Huber ${ }^{1}$
}

\begin{abstract}
Pichler Hefti, Jacqueline, Denise Sonntag, Urs Hefti, Lorenz Risch, Otto D. Schoch, Alexander J. Turk, Thomas Hess, Konrad E Bloch, Marco Maggiorini, Tobias M. Merz, Klaus M. Weinberger, and Andreas R. Huber. Oxidative stress in hypobaric hypoxia and influence on vessel-tone modifying mediators. High Alt Med Biol. 14:273-279, 2013.-Increased pulmonary artery pressure is a well-known phenomenon of hypoxia and is seen in patients with chronic pulmonary diseases, and also in mountaineers on high altitude expedition. Different mediators are known to regulate pulmonary artery vessel tone. However, exact mechanisms are not fully understood and a multimodal process consisting of a whole panel of mediators is supposed to cause pulmonary artery vasoconstriction.

We hypothesized that increased hypoxemia is associated with an increase in vasoconstrictive mediators and decrease of vasodilatators leading to a vasoconstrictive net effect. Furthermore, we suggested oxidative stress being partly involved in changement of these parameters.

Oxygen saturation $\left(\mathrm{SaO}_{2}\right)$ and clinical parameters were assessed in 34 volunteers before and during a Swiss research expedition to Mount Muztagh Ata $(7549 \mathrm{~m})$ in Western China. Blood samples were taken at four different sites up to an altitude of $6865 \mathrm{~m}$.

A mass spectrometry-based targeted metabolomic platform was used to detect multiple parameters, and revealed functional impairment of enzymes that require oxidation-sensitive cofactors. Specifically, the tetrahydrobiopterin (BH4)-dependent enzyme nitric oxide synthase (NOS) showed significantly lower activities (citrulline-to-arginine ratio decreased from baseline median 0.21 to 0.14 at $6265 \mathrm{~m}$ ), indicating lower NO availability resulting in less vasodilatative activity. Correspondingly, an increase in systemic oxidative stress was found with a significant increase of the percentage of methionine sulfoxide from a median $6 \%$ under normoxic condition to a median level of 30\% $(p<0.001)$ in camp 1 at $5533 \mathrm{~m}$. Furthermore, significant increase in vasoconstrictive mediators (e.g., tryptophan, serotonin, and peroxidation-sensitive lipids) were found.

During ascent up to $6865 \mathrm{~m}$, significant altitude-dependent changes in multiple vessel-tone modifying mediators with excess in vasoconstrictive metabolites could be demonstrated. These changes, as well as highly significant increase in systemic oxidative stress, may be predictive for increase in acute mountain sickness score and changes in $\mathrm{SaO}_{2}$.
\end{abstract}

Key Words: hypobaric hypoxia; hypoxic pulmonary vasoconstriction; nitric oxide; oxidative stress

\footnotetext{
${ }^{1}$ Center of Laboratory Medicine, Cantonal Hospital Aarau and University of Bern, Bern, Switzerland.

Divisions of ${ }^{2}$ Pneumology and ${ }^{10}$ Intensive Care Medicine, Inselspital Bern, University Hospital Bern, Bern, Switzerland.

${ }^{3}$ Biocrates Life Sciences AG, Innsbruck, Austria.

${ }^{4}$ Swiss Sports Clinic, Bern, Switzerland.

${ }^{5}$ Division of Clinical Biochemistry, Medical University Innsbruck, Innsbruck, Austria.

${ }^{6}$ Division of Pneumology, Department of Internal Medicine, Cantonal Hospital St. Gallen, St. Gallen, Switzerland.

${ }^{7}$ Division of Pneumology, and ${ }^{9}$ Medical Intensive Care Unit, University Hospital Zurich, Zurich, Switzerland.

${ }^{8}$ Division of Pneumology, Department of Internal Medicine, Cantonal Hospital Winterthur, Winterthur, Switzerland.
} 


\section{Introduction}

$\mathbf{H}$ GH ALTITUDE STAY MAY LEAD to acute mountain sickness (AMS), high altitude cerebral edema (HACE), and high altitude pulmonary edema (HAPE). Under hypoxic conditions, physiologically appearing pulmonary vasoconstriction is overwhelming in subjects with HAPE, suggesting exaggerated hypoxic pulmonary vasoconstriction (HPV) being the main trigger for developing HAPE (Maggiorini et al., 2001). Different metabolic pathways have been suggested to cause HPV and pulmonary hypertension.

The nitric oxide (NO) pathway is assumed to be one of the key regulators of the pulmonary artery vessel tone. Diminished bioavailability of the vasorelaxant NO may appear directly through reactive oxygen species (ROS), or secondary due to dysregulation of the underlying L-arginine metabolism, or diminished expression or activation of NOS (Forstermann, 2010). Symmetric and asymmetric dimethylarginine (SDMA and ADMA), which are known as a cardiovascular risk factors, compete with L-arginine for transportation into cells, thus reducing the substrate for NO synthesis (Vallance et al., 1992; MacAllister et al., 1996; Lluch et al., 2006). Furthermore, arachidonic and linoleic acids products (e.g., hydroxy-eicosatetranoic acids (HETE), prostaglandins, and leukotrienes) are determinants in the regulation of the vascular tone and possibly contribute to pulmonary hypertension (Ma et al. 2011; Chawengsub et al., 2009). In particular, serotonin has been shown to increase pulmonary artery pressure in hypoxia (Morecroft et al., 2007), partially in consequence of ROS-dependent generation of the transporter, providing serotonin to the site of action, has been demonstrated (Lawrie et al., 2005).

ROS may be involved in development of HPV and pulmonary hypertension due to diverse mechanisms, either by the above mentioned disruption in the NO-pathway, by interaction with the endothelial layer, or by uncoupling of oxidases, such as xanthine oxidase and nicotinamide adenine dinucleotide phosphate oxidase (Demarco et al., 2010 ; Jerkic et al., 2011 ; Lane et al., 2011). Because of their short half lives, quantification of ROS is difficult and requires special methodological settings. Nevertheless, the biochemical consequences of ROS can be readily detected by measuring the levels of intermediary metabolites altered by oxidative stress. These intermediate metabolites include polyunsaturated fatty acids (PUFA), such as linoleic acid or arachidonic acid, which can be oxidized to hydroxy-octadecadienoic acid (HODE) or hydroxy-eicosatetraenoic acid (HETE), respectively. Furthermore, under oxidative stress, prostaglandins and thromboxanes are produced from arachidonic acid over cyclooxygenase (Yoshida et al., 2006; Niki, 2008). In addition, amino acids such as methionine and tyrosine are oxidized to methionine sulfoxide (Vogt, 1995) and changes in enzyme products depending on ROS-sensitive cofactors may be used as markers of oxidative stress.

High altitude stay serves as a valuable model to investigate isolated effects of hypoxia, without the limitations of the most clinical studies investigating conditions with severe hypoxemia (e.g., critically ill patients), where there are many confounding factors such as multiple co-morbidities. Furthermore, most of our knowledge results from in vitro or animal studies, which might not reflect the real human pathway changes. We, therefore, hypothesized that progressive hypobaric hypoxia leads to changes in multiple vessel tone-modifying mediators with increase in metabolites with vasoconstrictive properties, paralleled by a decrease in metabolites with vasodilatatory effects, and that some of these pathway changes might be induced primarily by oxidative stress (Goodacre et al., 2004; Wishart et al., 2007; Gomase et al., 2008).

\section{Methods}

\section{Study participants}

Thirty-four healthy volunteers, aged between 29 and 65 years (mean 46 years), including 7 female participants, were examined during a high altitude expedition in 2005 to Mount Muztagh Ata $(7545 \mathrm{~m})$ in Western China. The design of the expedition was such that no strenuous physical exercise was necessary to accomplish the route. During the expedition, ample resting days allowing for good adaptation were scheduled to reduce the variables tested to those primarily involving hypobaric hypoxia rather than those due to exhausting exercise or exposure to heat or cold. The study was approved by the cantonal ethics board of Zurich (KEK-Nr. 1189 ) and is in accordance with the Declaration of Helsinki. All participants provided written informed consent.

\section{Study design and blood samples}

Medical testing, including blood sampling, clinical examination and assessment of AMS-scores, was performed first in Zürich at $490 \mathrm{~m} / 1474 \mathrm{ft}$ (baseline examination). After air travel to Islamabad, Pakistan $(500 \mathrm{~m})$, the journey continued by bus to Subash, China $(3730 \mathrm{~m})$, where the expedition started (day 1). The examinations were repeated at base camp $(4497 \mathrm{~m}$ above sea level) on day 3 , at camp 1 (5533 m) on day 6 , at camp $2(6265 \mathrm{~m})$ on day 13 , and at camp $3(6865 \mathrm{~m})$ on day 19 . To determine AMS, different scoring systems, such as the Lake Louise Score (LLS) and the environmental symptoms questionnaire cerebral score (AMS-C), were used (Roach, 1993).

We used 20-gauge butterfly cannulas (Becton Dickinson, Basel, Switzerland) and sterile tubes (Sarstedt AG, Sevelen, Switzerland) for collecting serum and citrated plasma. The blood drawings were done within $10 \mathrm{~min}$ in a standardized fashion. After centrifugation at $2300 \mathrm{~g}$ at the site (Rotanta, Hettich AG, Bäch, Switzerland), the supernatants were aliquoted into prelabeled tubes and immediately frozen at $\leq-20^{\circ} \mathrm{C}$ in a Peltier-element freezer. The samples were kept frozen until analysis in the laboratories.

\section{Laboratory testing}

AbsoluteIDQ ${ }^{\mathrm{TM}}$ p150 KIT plates (Biocrates Life Sciences AG, Innsbruck, Austria) were used according to the manufacturer's instructions for quantifying amino acids, acylcarnitines, phosphatidylcholines, hexoses, and biogenic amines (e.g., dimethylarginine, serotonin) using an API 4000 QTrap mass spectrometer (AB Sciex, Toronto) with electrospray ionization. After extraction, prostaglandins and other PUFA were analyzed by reverse phase HPLC-ESI-MS/MS in negative MRM detection mode using an API 4000 QTrap mass spectrometer (WO/2008/145384). Three groups of PUFA products were analyzed: the ROS-generated (i.e., nonenzymatic, linoleic acid derivatives $( \pm$ )9-hydroxy-10E,12Z-octadecadienoic acid [9-HODE], and 13(S)-hydroxy-9Z,11E-octadecadienoic acid [13S-HODE]), the arachidonic acid-derived lipoxygenase products (12(S)-hydroxy-5Z,8Z,10E,14Z-eicosatetraenoic acid 
[12S-HETE], 15(S)- hydroperoxy-5Z,8Z,11Z,13E-eicosatetraenoic acid [15S-HpETE], and 15(S)-hydroxy-5Z,8Z,11Z,13Eeicosatetraenoic acid [15S-HETE]), and the cyclooxygenase products (prostaglandins D2 and E2 [PGD2 and PGE2] and leukotriene B4 [LTB4]). C- reactive protein (CRP) concentrations in plasma were determined on a Dimenson Vista Analyzer 1500 (Siemens, Marburg, Germany) using a routine turbidimetric immunoassay. Osmolality measurements were performed on a routine freezing point osmometer OsmoLab One/16S (LLA Instruments, Berlin, Germany) in a standardized fashion (ISO15189).

\section{Statistical methods}

The data were assessed by a principal component analysis and a discriminant analysis incorporated in MarkerView Software (AB Sciex, Toronto). The dataset used for this study has been chosen due to its relevance to the topic and not due to a statistical procedure. Therefore, analysis was done using a Friedman repeated measures ANOVA on ranks and MannWhitney tests. Statistical software R version 2.8.1, SPSS Statistics, Version 17.0 (IBM, USA), as well as MetIDQ Stat Pack (Biocrates Life Sciences AG, Innsbruck, Austria) and GraphPad Prism (GraphPad Software, La Jolla, CA). Results are expressed as the median with interquartile range, and all box plots were drawn using Sigma Plot software version 10 with standard settings.

\section{Results}

\section{Course of the expedition and standard} laboratory parameters

Nineteen of 34 participants reached the summit of Mount Muztagh Ata $(7545 \mathrm{~m})$, which corresponds to a quite good success rate of $55.8 \%$. Mild to moderate AMS, corresponding to a AMS score $>3$ was seen in $8 / 34$ at base camp, in $9 / 34$ at camp 1 , in $9 / 33$ at camp 2 , and in $2 / 18$ at camp 3 . In camp 2 and 3 , the study population was reduced due to premature return, either because of more severe AMS symptoms or because of exhaustion. Additionally, blood sampling was limited at camp 3 because of adverse weather conditions. AMS scores increased with altitude, whereas $\mathrm{SaO}_{2}$ decreased over the altitude and time (Table 1).

During routine laboratory testing of the samples, no hemolysis or clots were found, and osmolalities were within the normal range (280-310 mosm/L, data not shown) were found. Samples were also tested for lipemia or icteria and were found to be negative. The sample quality could be fur- ther reassured by the good correlation of creatinine levels measured with a Jaffe-based method on a routine analyzer compared with creatinine results obtained by mass-spectrometry. Increase in lipoxygenase products, paralleled by low levels of cycloxygenase products over the altitude, are consistent with the broad use of nonsteroidal anti-inflammatory drugs and also reflect good preanalytic quality. Routine laboratory testing revealed a mild inflammatory response with increase in median CRP values from $0.4 \mathrm{mg} / \mathrm{L}$, interquartile range (0.4-1.0) at base line to $9.8 \mathrm{mg} / \mathrm{L}(4.1-22.3)$ at base camp, to $7.5 \mathrm{mg} / \mathrm{L}$ (3.4-20.0) at camp 1, 2.2 mg/L (1.5-84.2) at camp 2, and $1.9 \mathrm{mg} / \mathrm{L}(1.5-2.7)$ at camp 3. Lactate levels were only mildly elevated ( $<2.5 \mathrm{mmol} / \mathrm{L})$ (data not shown).

\section{Oxidative parameters}

Lipid oxidation. In normoxic serum, only 15S-HETE was present in quantifiable amounts, while the highly reactive hydroperoxide intermediate 15S-HpETE was below the limit of detection. All lipid peroxidation products were significantly increased under hypobaric conditions $(p<0.001)$. Figure 1 shows that PUFA increase up to a height of $5500 \mathrm{~m}$ and level off thereafter.

Methionine sulfoxidation. The amount of methionine sulfoxide (MetSO) determined in relation to methionine, a surrogate marker for oxidative stress, increased significantly from median $6 \%$ under normoxic conditions to $15 \%$ at base camp ( $4497 \mathrm{~m}$ ) and peaked at $30 \%$ under hypobaric conditions in camp 1 (5533 m; $p<0.001)$. With further ascent, the median values decreased but remained high $(20 \%$ methionine sulfoxidation) (Fig. 2).

Tetrahydrobiopterin-dependent enzyme activities. At baseline examination, the median phenylalanine plasma concentration was $84 \mu \mathrm{mol} / \mathrm{L}$ (75-98), and the median tyrosine concentration was $68 \mu \mathrm{mol} / \mathrm{L}(51-86)$, resulting in a tyrosine-to-phenylalanine ratio of 0.81 (0.70-0.91). At high altitudes, the median phenylalanine concentration increased significantly to $115 \mu \mathrm{mol} / \mathrm{L}$ (97-132) at base camp (4497 m), and with further climbing, reached $125 \mu \mathrm{mol} / \mathrm{L}$ (111-145) at $6865 \mathrm{~m}$. Conversely, median tyrosine plasma concentrations remained nearly constant or slightly decreased, up to an altitude of $5533 \mathrm{~m}$. In camps $2(6265 \mathrm{~m})$ and $3(6865 \mathrm{~m})$, tyrosine increased slightly to $78 \mu \mathrm{mol} / \mathrm{L}(62-102)$ and $87 \mu \mathrm{mol} / \mathrm{L}$ (76103), respectively. The different altitude-dependent concentration changes of the two amino acids were reflected by significantly lowered tyrosine-to-phenylalanine ratios. The

Table 1. Data Supply for Expedition Course and Incidence and Severity of Acute Mountain Sickness

\begin{tabular}{|c|c|c|c|c|c|}
\hline & Base line & Base camp & Camp 1 & Camp 2 & Camp3 \\
\hline Altitude (m) & 490 & 4497 & 5533 & 6265 & 6865 \\
\hline Day & & 3 & 6 & 13 & 19 \\
\hline Subjects included & $34 / 34$ & $34 / 34$ & $34 / 34$ & $33 / 34$ & $18 / 34$ \\
\hline $\begin{array}{l}\text { Subjects with acute } \\
\text { mountain sickness }\end{array}$ & $0 / 34(0 \%)$ & $8 / 34(23.5 \%)$ & $9 / 34(26.5 \%)$ & $9 / 33(27.3 \%)$ & $2 / 18(11.1 \%)$ \\
\hline AMS Score, median & 0 & 2 & 2 & 1 & 1 \\
\hline AMS Score, $\min$ & 0 & 0 & 0 & 0 & 0 \\
\hline AMS Score, $\max$ & 0 & 5 & 5 & 7 & 4 \\
\hline $\mathrm{SaO}_{2}$, mean & $99.6 \%$ & $83.9 \%$ & $75.5 \%$ & $73.3 \%$ & $74.5 \%$ \\
\hline$P$ values & & $<0.001$ & $<0.001$ & $<0.001$ & $<0.001$ \\
\hline
\end{tabular}




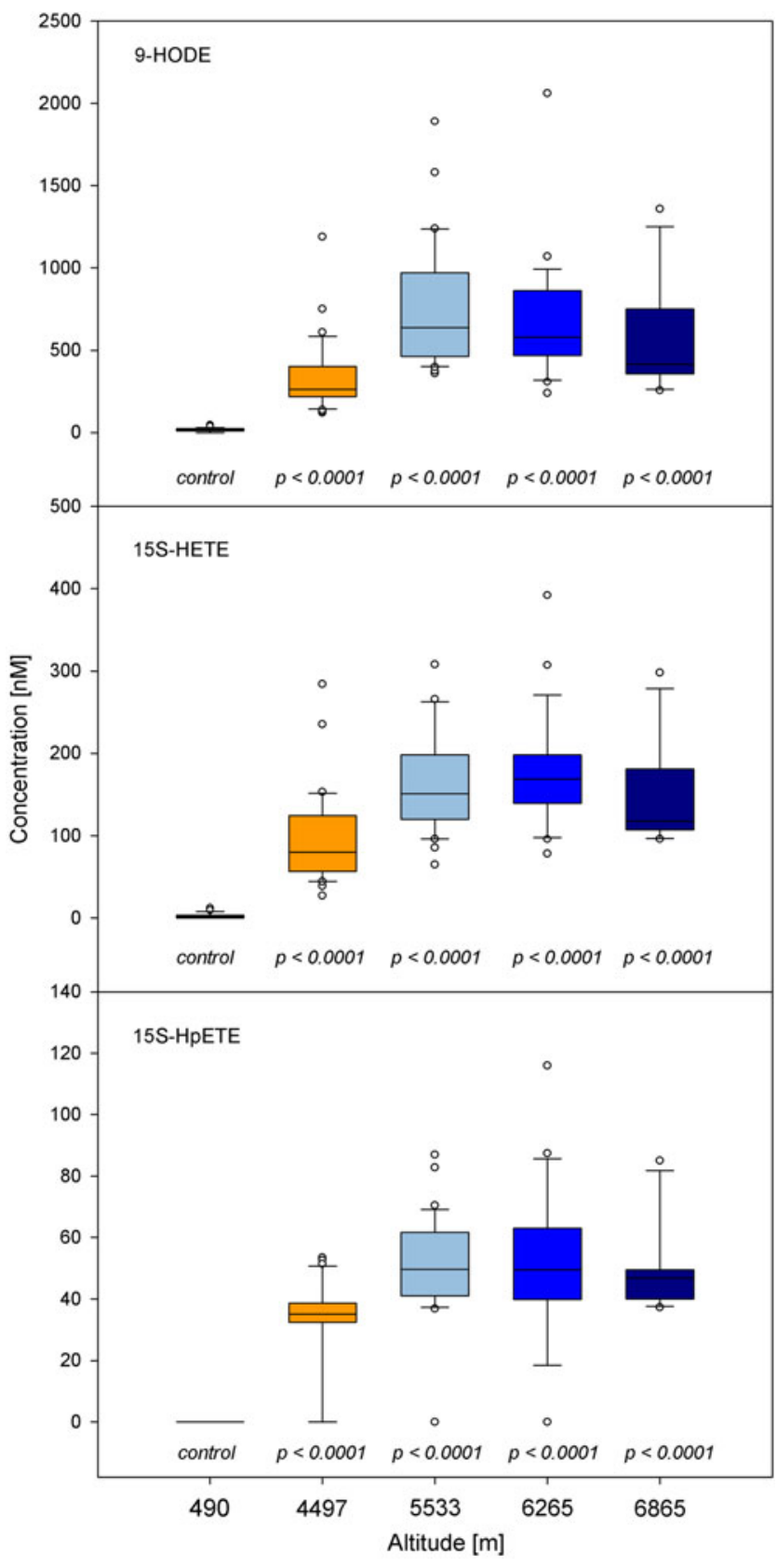

FIG. 1. Increasing PUFA (per-)oxidation at high altitude. Even the highly reactive, short-lived 15S-HpETE could be detected at significantly elevated concentrations.

ratio decreased to $0.62(0.55-0.68)$ at $4497 \mathrm{~m}$ and 0.55 $(0.50-0.64)$ at $5533 \mathrm{~m}$ above sea level. With further climbing, tyrosine-to-phenylalanine ratios increased again, but did not return to base level. Under hypobaric conditions, the citrulline-to-arginine ratio decreased significantly reflecting reduced NO generation (Fig. 3).

Symmetric and asymmetric dimethylarginine. The levels of ADMA did not significantly change between the different heights (data not shown). However, the concentrations of SDMA exhibited significant changes $(p=0.023): 0.39 \mu \mathrm{mol} / \mathrm{L}$ $(0.0-0.53)$ before expedition, $0.61 \mu \mathrm{mol} / \mathrm{L}(0.34-0.69)$ at base camp, $0.60 \mu \mathrm{mol} / \mathrm{L}(0.09-0.76)$ at camp $1,0.69 \mu \mathrm{mol} / \mathrm{L}(0.10$ $0.84)$ at camp 2, and $0.65 \mu \mathrm{mol} / 1(0.38-0.80)$ at camp 3.

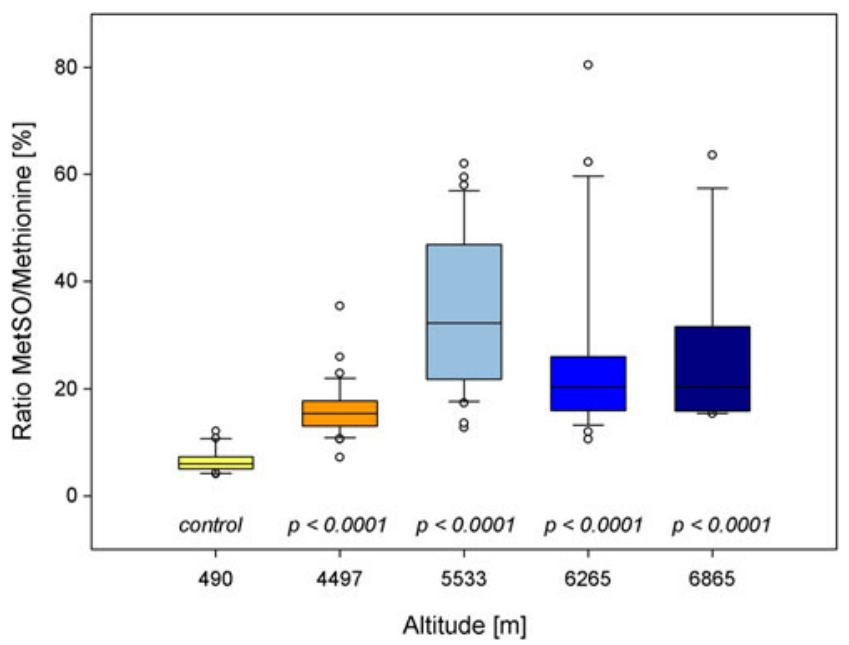

FIG. 2. Increased methionine sulfoxidation, given as the ratio of methionine sulfoxide to methionine (MetSO/Met), as a lipid-independent marker of oxidative stress.

Tryptophan and serotonin. The levels of tryptophan exhibited significant changes $(p<0.001)$ : median value $65 \mu \mathrm{mol} / \mathrm{L}$ (55-72) before expedition, $73 \mu \mathrm{mol} / \mathrm{L}(58-84)$ at base camp, $72 \mu \mathrm{mol} / \mathrm{L}(59-86)$ at camp 1, $85 \mu \mathrm{mol} / \mathrm{L} \mathrm{(69-105)} \mathrm{at} \mathrm{camp} \mathrm{2,}$ and $108 \mu \mathrm{mol} / \mathrm{L}(71-129)$ at camp 3 . The concentrations of serotonin also showed significant changes $(p=0.001)$ : median value $0.68 \mu \mathrm{mol} / \mathrm{L}(0.49-0.96)$ before expedition, $0.78 \mu \mathrm{mol} / \mathrm{L}$ (0.69-1.21) at base camp, $0.94 \mu \mathrm{mol} / \mathrm{L}(0.51-1.25)$ at camp 1 , $1.10 \mu \mathrm{mol} / \mathrm{L}(0.57-1.64)$ at camp 2, and $1.10 \mu \mathrm{mol} / \mathrm{L}(0.69-$ 1.38) at camp 3 (Fig. 4).

\section{Clinical parameters in correlation with marker of oxidative stress and vessel-tone modifying mediators}

Regression analysis was done for clinical parameters such as AMS-score and $\mathrm{SaO} 2$ with above mentioned parameters. Levels of MetSO, citrulline-to-arginine ratio, 15S-HpETE, 9HODE, and 13S-HODE appeared to be significant predictors for the level of AMS score obtained, as well as for changes in $\mathrm{SaO}_{2}$ (Fig. 5). In contrast, regression analysis did not show a significant result for other parameters, for example, serotonin, tryptophan, ADMA, or SDMA (results not shown).

\section{Discussion}

The most potent vasodilator NO results from the conversion of arginine to citrulline. The quantitative assessment of the product-to-substrate ratios such as tyrosine-to-phenylalanine (for PAH) and citrulline-to-arginine (for NOS) is useful to measure NO generation. For PAH and for NOS tetrahydropterin (BH4), an essential cofactor is needed. As BH4 is quickly oxidized under hypoxic conditions, it is no longer available at appropriate conditions to maintain the activity of $\mathrm{PAH}$ and NOS. The decreased citrulline-to-arginine ratio found in this study therefore reflects diminished endogenous NO generation, which might be explained by increase in oxidative stress with increasing altitude. Beside this indicator of oxidative stress, methionine sulfoxidation serves as a lipidindependent indicator of oxidative stress. The values of methionine sulfoxide obtained in the present study were even higher than those found under severe clinical conditions. Patients with septicemia and end stage renal disease (ESRD) 

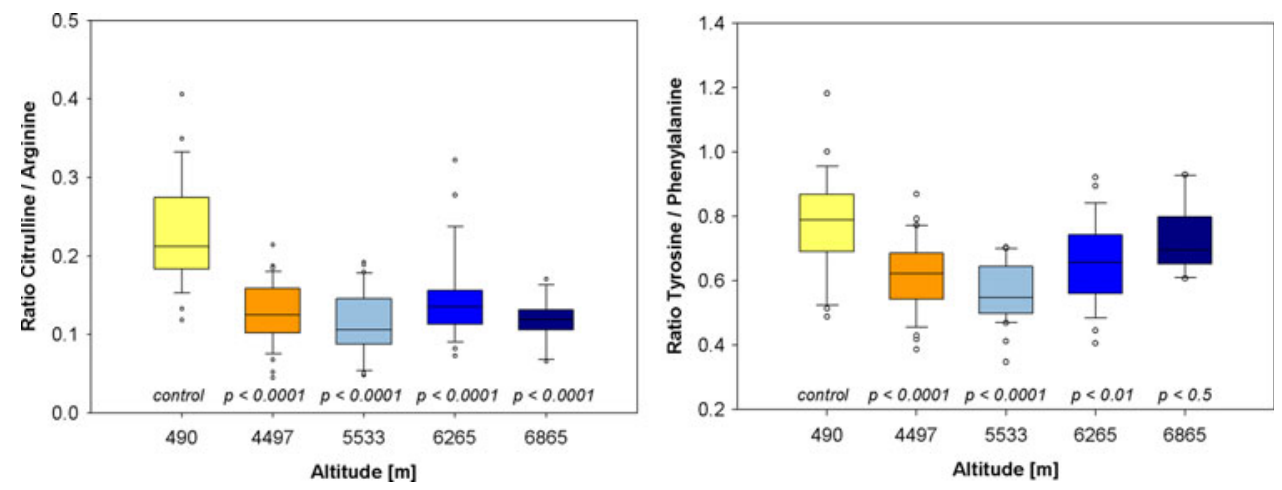

FIG. 3. Inhibition of BH4-dependent enzymes as a consequence of oxidative stress (NO synthase, left, and phenylalanine hydroxylase, right). Decrease in the citrulline-to-arginine ratio signifies diminished NO availability.

were characterized by elevated oxidative stress levels, with methionine sulfoxidation reaching a median of $16 \%$ in ESRD (WO 2010/139341) and a 20\% in a mixed sepsis cohort (unpublished data) compared to our findings with significant higher median levels of $30 \%$ at camp 1 (5,533 m). Together with the increase of PUFA oxidation products (i.e., ROSgenerated linoleic acid derivates and arachidonic acidproducts), these results strongly suggest relevant systemic generation of ROS in vivo.

There is controversy regarding whether ROS contribute to high altitude-related diseases, especially HAPE and HACE

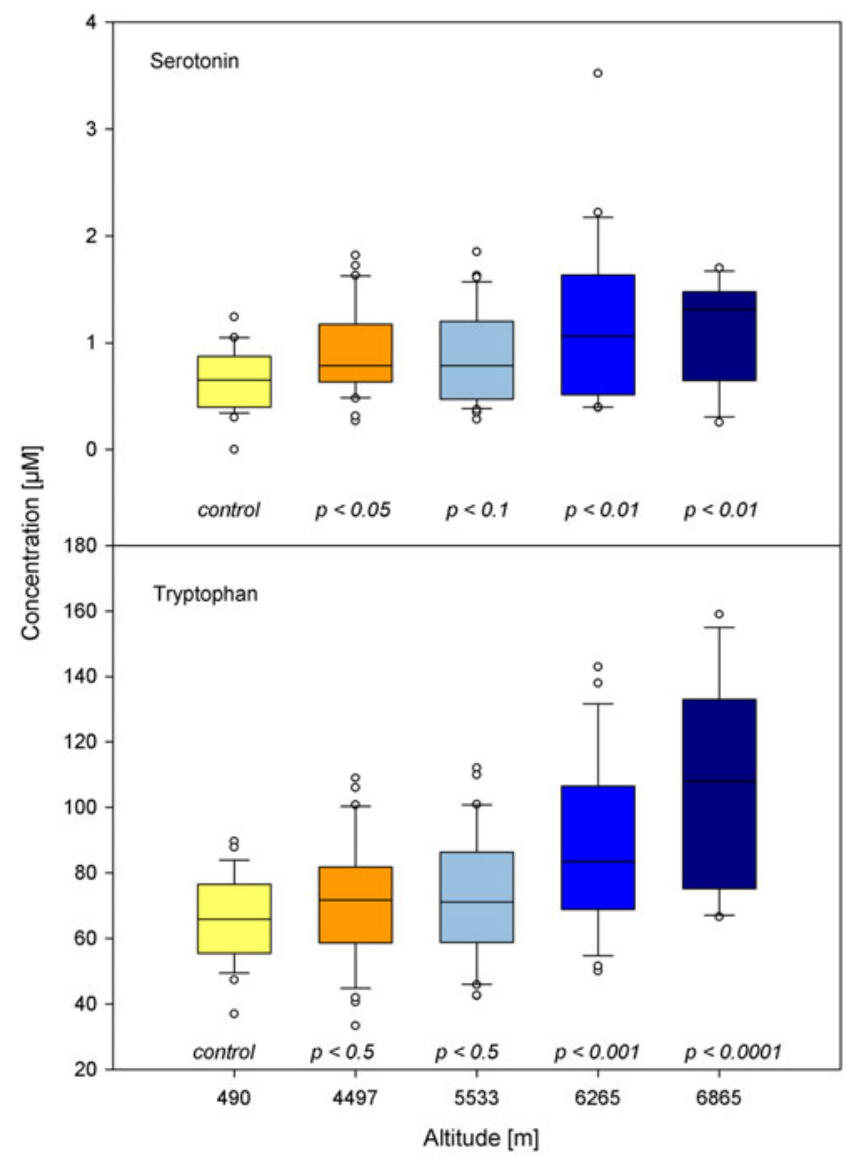

FIG. 4. Significant increase in serotonin and, more pronounced, in its precursor tryptophan, which are both known to have vasoconstrictive properties.
(Araneda et al., 2005; Bailey et al., 2009). It is known that ROS directly increases vascular permeability (Lee et al., 2006). As shown, our results suggest ROS-dependent decrease in $\mathrm{NO}$ availability under hypobaric hypoxia. Of course, other mediators could be involved as well. In agreement, other studies have shown that a lack of $\mathrm{NO}$ (which has vasorelaxant properties in the pulmonary arteries) may also contribute to endothelial dysfunction, especially in pulmonary hypertension-induced HAPE (Bailey et al., 2010). In addition, we found significant increase in ROS-dependent 15-HETE and 12HETE, which both are known to have vasoconstrictive properties (Burhop et al., 1988; Nishiyama et al., 1998; Yiu et al., 2003). The essential amino acid tryptophan, the biogenic amines serotonin, and SDMA, which all are supposed to have vasoconstrictive function in pulmonary vessels, also increased significantly during the high altitude stay. This pattern of metabolites may be partially responsible for the development of physiological hypoxic pulmonary vasoconstriction.

$\mathrm{SaO}_{2}$ decreases with reduced barometric pressure by reduced oxygen tension pressure. $\mathrm{SaO}_{2}$ might have a more pronounced decline as HPV leads to increased ventilation/ perfusion mismatch. This hypothesis is supported by the results of the regression analysis, pursuant to which metabolites with potential net-vasoconctricitve effect (e.g., MetSO, citrulline-to-arginine ratio, 15S-HpETE, and 13-HODE) showed to be possible predictors for changes in AMS score and $\mathrm{SaO} 2$ (Fig. 5). Although it is well accepted that (hypobaric) hypoxia leads to increase in pulmonary artery pressure, further investigations are needed to clarify whether these findings not only correlate but also are causative with increase in pulmonary artery pressure or development of HAPE (Duplain et al., 2000; Sylvester et al., 2012). As prolonged exposure to hypobaric hypoxia serves as a pathophysiologic model in critically ill patients or patients with pulmonary diseases, additional investigations are needed to clarify relevance of ROS and examined mediators in disease progression or extensive hypoxic pulmonary vasoconstriction. Moreover, the potential role of ROS-targeted therapy in the prevention of hypoxic pulmonary vasoconstriction and pulmonary hypertension needs further attention.

The main limitation of this study is the lack of surrogate markers of pulmonary vasoconstriction (e.g., echocardiographic and pressure measurements). The relatively small cohort size, although from our knowledge this is the biggest cohort size in the setting of a high altitude expedition where 

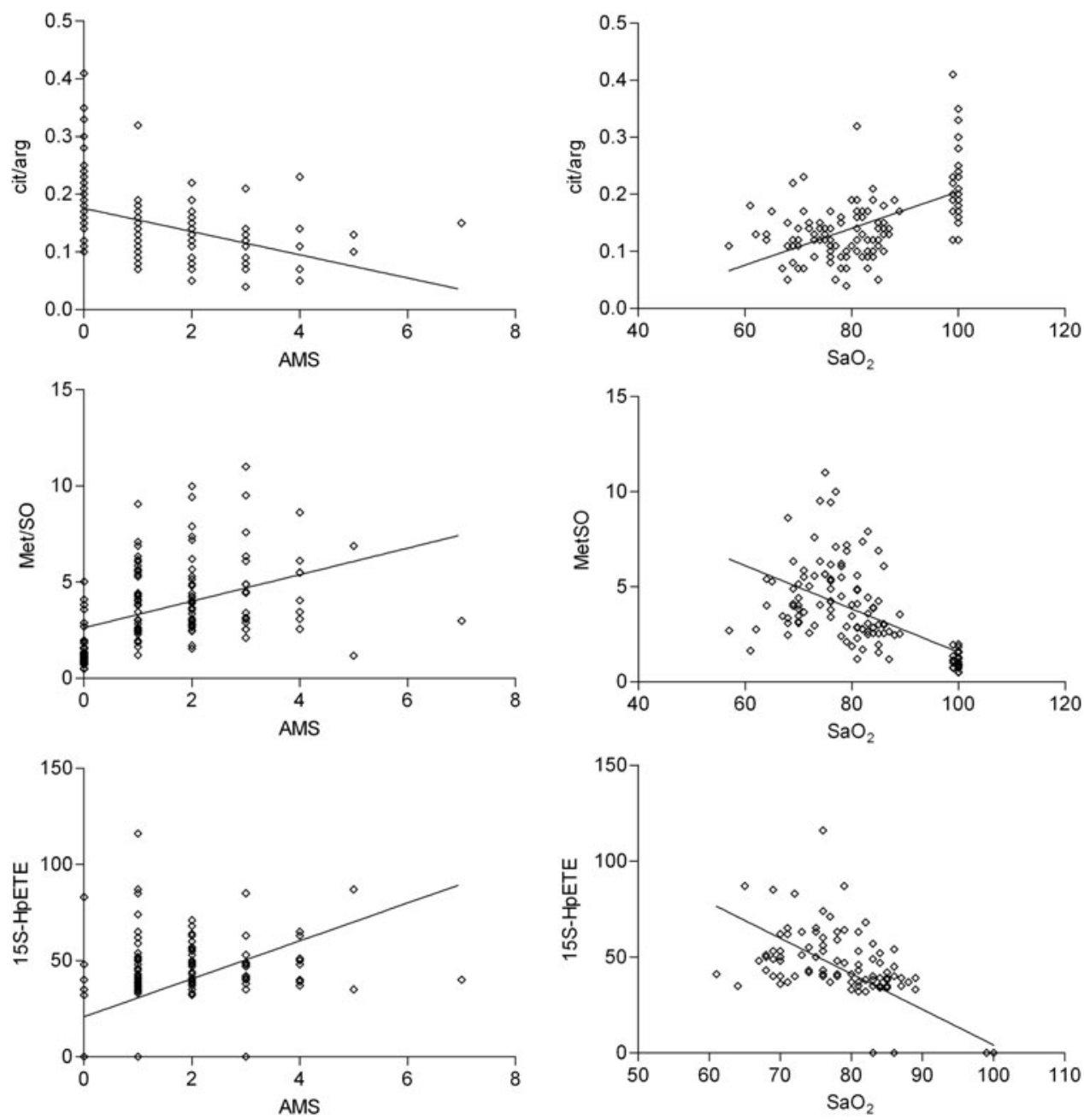

FIG. 5. Regression analysis of clinical parameters compared with oxidative stress related parameters. For AMS: MetSO ( $\beta$ coefficient $=0.41, \mathrm{R} 2=0.17, p<0.001)$, Cit $/ \operatorname{Arg}(\beta$-coefficient $=-0.43, \mathrm{R} 2=0.18, p<0.001), 15 \mathrm{~S}$-HpETE $(\beta$-coefficient $=0.53$, $\mathrm{R} 2=0.28, p<0.001)$. For $\mathrm{SaO}_{2}$ : MetSO $(\beta$-coefficient $=-0.58, \mathrm{R} 2=0.34, p<0.001)$, Cit $/ \operatorname{Arg}(\beta$-coefficient $=0.57, \mathrm{R} 2=0.32$, $p<0.001)$, 15S-HpETE $(\beta$-coefficient $=-0.81, \mathrm{R} 2=0.66, p<0.001)$.

measurements have been done up to $6865 \mathrm{~m}$, limits the final conclusion. The selection of the fittest and least affected subjects in the higher camps might explain the recurrent pattern of metabolic changes with leveling off over the altitude and time. This statement is supported by the recovery of $\mathrm{SaO}_{2}$ at camp 3 and by the relatively low percentage of AMS-affected subjects at higher altitude (Table1). Therefore, statements on how severe AMS or HAPE are influenced by oxidative stress are sparse. The results reflect more the usual adaption to hypobaric hypoxia, as none of the subjects suffered from severe AMS. In respect to the limited amount of blood samples that can be drawn, the difficult and complicated research setting during a high altitude expedition, and the multiple pathways that may be involved in the development of hypoxic pulmonary vasoconstriction, mass spectrometry-based analysis of metabolic changes offers a specific and sensitive approach and further enables to detect possible escape phenomenons.

This field study is the first study focusing on oxidative stress-related changes with a multi-level approach in humans at extreme altitude. Our results show multiple, significant altitude-dependent changes in hypoxia-related metabolites with domination of vasoconstrictive net effect. Our results suggest increased concentration of vasoconstrictive metabo- lites being associated with increase in AMS score and worsened oxygenation. This novel approach extends the understanding of hypoxia-related mechanisms on a biochemical level. Further studies are required to confirm vascular dysregulation as a result of biochemical pathway alteration shown.

In conclusion, we describe the effects of hypobaric hypoxia during high altitude trekking and mountaineering. Our results suggest pronounced systemic oxidative stress under hypoxic conditions being correlated with changes in AMS score and decreased $\mathrm{SaO}_{2}$. In addition, we demonstrate significant changes in multiple biochemical pathways with vasoconstrictive properties. This novel approach extends the understanding of hypoxia-related mechanisms on a biochemical level. Further studies are required to confirm vascular dysregulation as a result of biochemical pathway alteration shown.

\section{Acknowledgments}

We are grateful to the 34 volunteers and all the members of the Swiss Muztagh Ata Expedition Team for their enthusiastic participation. Special thanks are due to the mountaineering 
guides from Kobler and partners and all the porters from Pakistan, China, and Nepal who made this expedition a memorable and safe one. The authors thank the Swiss Society of mountain medicine, the Swiss National Fund, and the Swiss lottery foundation State of Aargau for their financial support.

\section{Author Disclosure Statement}

No competing financial interests exist.

\section{References}

Araneda OF, Garcia C, Lagos N, et al. (2005). Lung oxidative stress as related to exercise and altitude. Lipid peroxidation evidence in exhaled breath condensate: a possible predictor of acute mountain sickness. Eur J Appl Physiol 95:383-390.

Bailey DM, Dehnert C, Luks AM, et al. (2010). High-altitude pulmonary hypertension is associated with a free radicalmediated reduction in pulmonary nitric oxide bioavailability. J Physiol 588:48374847.

Bailey DM, Evans KA, James PE, et al. (2009). Altered free radical metabolism in acute mountain sickness: Implications for dynamic cerebral autoregulation and blood-brain barrier function. J Physiol 587:73-85.

Burhop KE, Selig WM, and Malik AB. (1988). Monohydroxyeicosatetraenoic acids (5-HETE and 15-HETE) induce pulmonary vasoconstriction and edema. Circ Res 62:687-698.

Chawengsub Y, Gauthier KM, and Campbell WB. (2009). Role of arachidonic acid lipoxygenase metabolites in the regulation of vascular tone. Am J Physiol Heart Circ Physiol 297:H495-507.

Demarco VG, Whaley-Connell AT, Sowers JR, Habibi J, and Dellsperger KC. (2010). Contribution of oxidative stress to pulmonary arterial hypertension. World J Cardiol 2:316-324.

Duplain H, Sartori C, Lepori M, et al. (2000). Exhaled nitric oxide in high-altitude pulmonary edema: Role in the regulation of pulmonary vascular tone and evidence for a role against inflammation. Am J Respir Crit Care Med 162:221-224.

Forstermann U. (2010). Nitric oxide and oxidative stress in vascular disease. Pflugers Arch 459:923-939.

Gomase VS, Changbhale SS, Patil SA, and Kale KV. (2008). Metabolomics. Curr Drug Metab 9:89-98.

Goodacre R, Vaidyanathan S, Dunn WB, Harrigan GG, and Kell DB. (2004). Metabolomics by numbers: Acquiring and understanding global metabolite data. Trends Biotechnol 22:245-252.

Jerkic M, Kabir MG, Davies A, et al. (2011). Pulmonary hypertension in adult Alk1 heterozygous mice due to oxidative stress. Cardiovasc Res 92:375-384.

Lane KL, Talati M, Austin E, et al. (2011). Oxidative injury is a common consequence of BMPR2 mutations. Pulm Circ 1:72-83.

Lawrie A, Spiekerkoetter E, Martinez EC, et al. (2005). Interdependent serotonin transporter and receptor pathways regulate S100A4/Mts1, a gene associated with pulmonary vascular disease. Circ Res 97:227-235.
Lee KS, Kim SR, Park SJ, et al. (2006). Hydrogen peroxide induces vascular permeability via regulation of vascular endothelial growth factor. Am J Respir Cell Mol Biol 35:190-197.

Lluch P, Mauricio MD, Vila JM, et al. (2006). Accumulation of symmetric dimethylarginine in hepatorenal syndrome. Exp Biol Med (Maywood) 231:70-75.

Ma C, Li Y, Ma J, et al. (2011). Key role of 15-lipoxygenase/15hydroxyeicosatetraenoic acid in pulmonary vascular remodeling and vascular angiogenesis associated with hypoxic pulmonary hypertension. Hypertension 58:679-688.

MacAllister RJ, Parry H, Kimoto M, et al. (1996). Regulation of nitric oxide synthesis by dimethylarginine dimethylaminohydrolase. Br J Pharmacol 119:1533-1540.

Maggiorini M, Melot C, Pierre S, et al. (2001). High-altitude pulmonary edema is initially caused by an increase in capillary pressure. Circulation 103:2078-2083.

Morecroft I, Dempsie Y, Bader M, et al. (2007).Effect of tryptophan hydroxylase 1 deficiency on the development of hypoxiainduced pulmonary hypertension. Hypertension 49:232-236.

Niki E. (2008). Lipid peroxidation products as oxidative stress biomarkers. Biofactors 34:171-180.

Nishiyama M, Okamoto H, Watanabe T, et al. (1998). Endothelium is required for 12-hydroperoxyeicosatetraenoic acid-induced vasoconstriction. Eur J Pharmacol 341:57-63.

Sylvester JT, Shimoda LA, Aaronson PI, and Ward JP. (2012). Hypoxic pulmonary vasoconstriction. Physiol Rev 92:367-520.

Vallance P, Leone A, Calver A, Collier J, and Moncada S. (1992). Accumulation of an endogenous inhibitor of nitric oxide synthesis in chronic renal failure. Lancet 339:572-575.

Vogt W. (1995). Oxidation of methionyl residues in proteins: Tools, targets, and reversal. Free Radic Biol Med 18:93-105.

Wishart DS, Tzur D, Knox C, et al. (2007). HMDB: The Human Metabolome Database. Nucleic Acids Res 35:D521-526.

Yiu SS, Zhao X, Inscho EW, and Imig JD. (2003). 12-Hydroxyeicosatetraenoic acid participates in angiotensin II afferent arteriolar vasoconstriction by activating L-type calcium channels. J Lipid Res 44:2391-2399.

Yoshida Y, and Niki E. (2006). Bio-markers of lipid peroxidation in vivo: Hydroxyoctadecadienoic acid and hydroxycholesterol. Biofactors 27:195-202.

Address correspondence to: Prof. Andreas R. Huber Center of Laboratory Medicine Cantonal Hospital Aarau AG Tellstrasse 5001 Aarau Switzerland

E-mail: andreas.huber@ksa.ch

Received November 12, 2012; accepted in final form March 7, 2013 